\title{
MAXIMAL SUBSPACES OF BESOV SPACES INVARIANT UNDER MULTIPLICATION BY CHARACTERS ${ }^{1}$ \\ BY
}

R. JOHNSON

\begin{abstract}
Unlike the familiar $L^{p}$ spaces, neither the homogeneous Besov spaces nor the $H^{p}$ spaces, $0<p<1$, are closed under multiplication by the functions $x \rightarrow e^{i\langle x, h\rangle}$. We determine the maximal subspace of these spaces which are closed under multiplication by these functions, which are the characters of $R^{n}$.
\end{abstract}

In this paper we investigate the maximal subspaces of Besov spaces invariant under multiplication by characters. The usefulness of such a study is related to applications to multipliers. Translation invariant operators from $L^{p}\left(R^{n}\right)$ into $L^{q}\left(R^{n}\right)$ are generally characterized by two kinds of properties-smoothness properties and growth restrictions. The smoothness properties are most naturally expressed in terms of the Besov spaces. The convoluteurs from $H^{p}\left(R^{n}\right)$ into $H^{q}\left(R^{n}\right)$ for $0<p<1 \leqslant q \leqslant \infty$ satisfy smoothness conditions of positive orders which already imply growth conditions by the Sobolev theorem. In this case one finds $C v\left(H^{p}, H^{q}\right)=$ $\dot{B}_{q \infty}^{n(1 / p-1)}[10]$. The smoothness part of this result remains valid for $p>1$ but it no longer implies a growth condition and we should expect the inclusion $C v\left(H^{p}, H^{q}\right) \subset \dot{B}_{q \infty}^{n(1 / p-1)}$ to be a proper inclusion in this case. One approach to imposing growth conditions is to note that while elements of $\dot{B}_{q \infty}^{\alpha}$ may grow at infinity, they must grow in a regular way. If we ask that a distribution be in $\dot{B}_{q \infty}^{\alpha}$ after multiplying by any oscillating function such as $e^{i\langle x, h\rangle}$, we should eliminate the functions that grow at infinity. This leads to the problem that we pose in this paper: Characterize the distributions $T \in \dot{B}_{p q}^{\alpha}$ such that $e^{i\langle x, h\rangle} T \in \dot{B}_{p q}^{\alpha}$ for all $h \in R^{n}$. Since $C v\left(L^{p}, L^{q}\right)$ is known to be invariant under such multiplication [7], we improve the inclusion for translation invariant operators. It should also be noted that the subspaces defined in such a way have interesting functorial properties in relation to duality, interpolation and so forth. The behaviour we find is in marked contrast to the situation for the inhomogeneous Besov spaces, where $e^{i\langle x, h\rangle} T \in B_{p q}^{\alpha}$ for any $T \in B_{p q}^{\alpha}$. This follows from the results of Murata [14] and the fact that

Received by the editors November 14, 1977.

AMS (MOS) subject classifications (1970). Primary 46E35; Secondary 42A18, 42A68, 35K05.

( ${ }^{1}$ )This paper was partly prepared at the Mittag-Leffler Institute while the author was on leave from the University of Maryland, with financial support from the Mittag-Leffler Foundation. 
$e^{i\langle x, h\rangle} \in B_{\infty \infty}^{\alpha}$ for any $h \in R^{n}$ and any $\alpha$ real.

Since the Fourier transform maps the Besov spaces into spaces which generalize the convolution algebras of Beurling [5], [6] and sends multiplication by $e^{i\langle x, h\rangle}$ to translation by $h$, we will also study subspaces of these spaces invariant under translation. We give several applications, including an intrinsic characterization of inhomogeneous Besov spaces in terms of homogeneous Besov spaces, a characterization of the Fourier transform image of the Morrey spaces, the positive part of the solution to an old problem of Peetre's and a proof of the nonexistence of nonnegative elements of $\dot{B}_{p q}^{\alpha}$ for $\alpha$ sufficiently negative.

0. Notation. The notation will be standard. Define $L^{p}\left(R^{n}\right)$ in the usual way for $0<p<\infty$, and let $\phi \in \mathcal{S}$ be a rapidly decreasing function such that $\int \phi(x) d x=1$. Set $\phi_{t}(x)=t^{-n} \phi\left(x t^{-1}\right)$. Given a tempered distribution $f \in \mathcal{S}^{\prime}$, set

$$
u(x, t)=f * \phi_{t}=\left\langle f_{y}, \phi_{t}(x-y)\right\rangle
$$

and

$$
u^{+}(x)=\sup _{t>0}|u(x, t)| .
$$

It was shown in [4] that $f \in H^{p}\left(R^{n}\right), 0<p<\infty$, iff $u^{+} \in L^{p}\left(R^{n}\right)$ and $\|f\|_{H^{p}}=\left\|u^{+}\right\|_{L^{p}}, 0<p<\infty$. It is well known that $H^{p}$ is isomorphic to $L^{p}$ in the case $1<p<\infty$. The proper interpretation of $H^{\infty}$ is the space BMO which will be defined below.

Both homogeneous and inhomogeneous Besov spaces will be used. Let $\tau_{h}$ denote the translation operator and $\Delta_{h}$ the first difference operator, defined respectively by $\tau_{h} f(x)=f(x-h)$ and $\Delta_{h} f=\left(\tau_{-h}-I\right) f$. If $0<\alpha<1,1<$ $p, q<\infty, f \in \dot{B}_{p q}^{\alpha}$ iff $\left(\int\left(\left\|\Delta_{h} f\right\|_{p} /|h|^{\alpha}\right)^{q}|h|^{-n} d h\right)$ is finite (with the obvious interpretation if $p$ or $q=\infty$ ). For an arbitrary real number $\alpha$, the homogeneous Besov spaces are defined by the Riesz potentials. The Riesz potential of order $\beta$ is the closure of the operator defined on a dense subset of $\delta^{\prime}$ by

$$
R^{\beta_{f}}{ }^{\wedge}(\xi)=|\xi|^{-\beta} \hat{f}(\xi) .
$$

Here we take as the definition of the Fourier transform

$$
F f(\xi)=\hat{f}(\xi)=\int e^{-i\langle x, \xi\rangle} f(x) d x,
$$

for $f \in \mathcal{S}$, extended by duality to $\mathcal{S}^{\prime}$, and $x \in R^{n}, \xi \in R^{n},\langle x, \xi\rangle=x_{1} \xi_{1}$ $+\cdots+x_{n} \xi_{n}$. The definition of $\dot{B}_{p q}^{\alpha}$ for $\alpha$ arbitrary is accomplished by $R^{\beta} \dot{B}_{p q}^{\alpha}=\dot{B}_{p q}^{\alpha+\beta}$. There is no global restriction on the growth of an $f \in \dot{B}_{p q}^{\alpha}$; for $p=q=2$ and $\alpha$ nonnegative integral, this is the class of functions such that all their derivatives of order $\alpha$ are in $L^{2}$, with no condition on the function itself. The elements of $\dot{B}_{p q}^{\alpha}$ are actually equivalence classes modulo polynomials. 
The inhomogeneous Besov spaces are defined by imposing a global restriction on $f ; f \in B_{p q}^{\alpha}$ iff $f \in L^{p} \cap \dot{B}_{p q}^{\alpha}$ for $\alpha>0$, and for $\alpha<0$, the spaces are generated by the Bessel potentials

$$
J^{\beta} B_{p q}^{\alpha}=B_{p q}^{\alpha+\beta}
$$

where

$$
J^{\beta} f^{\wedge}(\xi)=\left(1+|\xi|^{2}\right)^{-\beta / 2} \hat{f}(\xi) .
$$

One of our main results will give an intrinsic characterization of $B_{p q}^{\alpha}$ in terms of $\dot{B}_{p q}^{\alpha}$ for $\alpha>0$.

The Fourier transform image of $\dot{B}_{p q}^{\alpha}$ is in $K_{p^{\prime} q}^{\alpha}$ if $1<p<2$, where we can define

$$
K_{p q}^{0}=\left\{f \mid f \text { measurable, }\left[\sum_{-\infty}^{\infty}\left(\int_{2^{k}<|y|<2^{k+1}}|f(y)|^{p} d y\right)^{q / p}\right]^{1 / q}<\infty\right\}
$$

(with the usual interpretations if $p$ or $q$ is $\infty$ ). For general $\alpha$, if $v(x)=b_{n}|x|^{n}$ is the volume of a ball of radius $|x|$ centered at 0 , we have $f \in K_{p q}^{\alpha}$ iff $f v^{\alpha / n} \in K_{p q}^{0}$. These spaces will be shown to be related to the Morrey-Stampacchia spaces $L^{p, \lambda}$. A measurable function belongs to $L^{p, \lambda}$ if for any $x \in R^{n}$, $r>0, \exists a_{x r}$ such that

$$
\int_{B(x, r)}|f(y)-a|^{p} d y<A r^{\lambda}
$$

where $B(x, r)$ is the ball of radius $r$ centered at $x$, and $A$ is independent of $x$ and $r$. The indices satisfy $1<p<\infty, 0<\lambda<n+p$. It is known that $L^{p, 0}=L^{p}\left(R^{n}\right), L^{p, n}$ is independent of $p$ and is the John-Nirenberg space BMO of functions of bounded mean oscillation, and for $n<\lambda<n+p$, $L^{p, \lambda}=\dot{B}_{\infty \infty}^{(\lambda-n) / p}$.

Our application of the results we derive will be to convoluteurs and we denote

$$
C v(X, Y)=\{K \mid f \in X, K * f \in Y \text { and }\|K * f\|<C\|f\|\},
$$

and for multipliers

$$
M(X, Y)=\{\hat{K} \mid K \in C v(X, Y)\}
$$

and generally

$$
F X=\{\hat{f} \mid f \in X\}
$$

so that

$$
M(X, Y)=F C v(X, Y)
$$


It should be noted that if a space $X$ is not invariant under multiplication by $e^{i\langle x, h\rangle}$, there is an obvious way to construct the maximal subspace of $X$ which is invariant by simply setting

$$
\lambda X=\left\{f \in X \mid e^{i\langle x, h\rangle} f \in X, \forall h \in R^{n}\right\} .
$$

However, without some idea of the behavior of the norm of $e^{i\langle x, h\rangle} f$ as a function of $h$, it cannot be asserted that $\lambda X$ is closed under the induced topology. On the basis of the behavior of the norm in familiar spaces, one expects that there will be a bound of the form $\left\|e^{i\langle x, h\rangle} f\right\|_{X} \leqslant C\|f\|_{X}$, with $C$ independent of $h$. This is not the case for all spaces, and we are forced to use alternative bounds. We thus make the following definitions: for $\alpha>0$,

$$
\begin{aligned}
\pi \dot{B}_{p q}^{\alpha}=\left\{f \in \dot{B}_{p q}^{\alpha} \mid e^{i\langle x, h\rangle} f \in \dot{B}_{p q}^{\alpha}, \quad \forall h \in R^{n},\right. \\
\text { with } \left.\dot{B}_{p q}^{\alpha}\left(e^{i\langle x, h\rangle} f\right) \leqslant C\left(A(f)|h|^{\alpha}+\dot{B}_{p q}^{\alpha}(f)\right)\right\},
\end{aligned}
$$

while for $\alpha \leqslant 0$, and for all other spaces $X$ considered in this paper,

$$
\begin{aligned}
& \pi \dot{B}_{p q}^{\alpha}=\left\{f \in \dot{B}_{p q}^{\alpha} \mid e^{i\langle x, h\rangle} f \in \dot{B}_{p q}^{\alpha},\right. \forall h \in R^{n}, \\
&\text { with } \left.\dot{B}_{p q}^{\alpha}\left(e^{i\langle x, h\rangle} f\right) \leqslant C \dot{B}_{p q}^{\alpha}(f)\right\}, \\
& \pi X=\left\{f \in X \mid e^{i\langle x, h\rangle} f \in X, \forall h \in R^{n},\left\|e^{i\langle x, h\rangle} f\right\|_{X} \leqslant C\|f\|_{X}\right\} .
\end{aligned}
$$

In this paper, invariance under translation is studied only for negative indices and hence we always consider

$$
\tau X=\left\{f \in X \mid \tau_{h} f \in X, \quad \forall h \in R^{n}, \quad\left\|\tau_{h} f\right\|_{X}<C\|f\|_{X}\right\} .
$$

1. Results for homogeneous Besov spaces. For $\alpha>0$ the property of invariance under $f \rightarrow f e^{i\langle x, h\rangle}$ characterizes the inhomogeneous Besov spaces as subsets of the homogeneous Besov spaces. For $\alpha<0$ this cannot be true since $\dot{B}_{p q}^{\alpha} \subseteq B_{p q}^{\alpha}$ if $\alpha<0$.

THEOREM 1.1. Let $\alpha>0,1 \leqslant p, q \leqslant \infty$. Then one has $\pi \dot{B}_{p q}^{\alpha}=B_{p q}^{\alpha}$.

PROof. The proof will be by induction on the integer $m$ such that $m<\alpha<$ $m+1$. Consider the case $0<\alpha<1$ and assume that $f \in B_{p q}^{\alpha}$ (the case $\alpha=1$ requires a separate argument). From the identity

$$
\Delta_{\eta}\left(f e^{i\langle x, h\rangle}\right)=\left(\Delta_{\eta} f\right) e^{i\langle x+\eta, h\rangle}+f(x) \Delta_{\eta}\left(e^{i\langle x, h\rangle}\right),
$$

it follows that

$$
\left|\Delta_{\eta}\left(f e^{i\langle x, h\rangle}\right)\right| \leqslant\left|\Delta_{\eta} f(x)\right|+A|f(x)| \min (|\eta||h|, 1) .
$$

We calculate the $\dot{B}_{p q}^{\alpha}$ norm of $e^{i\langle x, h\rangle} f$ by taking the $L^{p}$ norm of the above expression with respect to $x$, dividing by $|\eta|^{\alpha}$ and taking the $L^{q}$ norm of the resulting expression with respect to $\eta$ for the measure $|\eta|^{-n} d \eta$. We see then 
that

$$
\dot{B}_{p q}^{\alpha}\left(f e^{i\langle x, h\rangle}\right) \leqslant \dot{B}_{p q}^{\alpha}(f)+A\|f\|_{p}|h|^{\alpha} .
$$

When $1 \leqslant \alpha \leqslant 2$, the proof is more complicated. There are now three terms in the identity analogous to the one used in the case $0<\alpha<1$ :

$$
\begin{aligned}
\Delta_{\eta}^{2}\left(e^{i\langle x, h\rangle} f\right)(x)= & \left(\Delta_{\eta}^{2} f(x)\right) e^{i\langle\eta+x, h\rangle} \\
& +e^{i\langle x, h\rangle}(f(x)-f(x-\eta))\left(e^{i\langle\eta, h\rangle}-e^{-i\langle\eta, h\rangle}\right) \\
& +f(x) e^{i\langle x, h\rangle}\left(e^{i\langle\eta, h\rangle}+e^{-i\langle\eta, h\rangle}-2\right) .
\end{aligned}
$$

The estimate which follows from this is

$$
\begin{gathered}
\left\|\Delta_{\eta}^{2}\left(e^{i\langle x, h\rangle} f\right)\right\|_{p} \leqslant\left\|\Delta_{\eta}^{2} f\right\|_{p}+2\|f(\cdot-\eta)-f\|_{p}|\sin \langle\eta, h\rangle| \\
+2\|f\|_{p}|1-\cos \langle\eta, h\rangle| .
\end{gathered}
$$

If we calculate the $\dot{B}_{p q}^{\alpha}$ norm of $e^{i\langle x, h\rangle} f$ as above, the first term on the right-hand side gives us $\dot{B}_{p q}^{\alpha}(f)$. Since $|1-\cos x|<\min \left(\frac{1}{2} x^{2}, 2\right)$, the third term is dominated by $\|f\|_{p}|h|^{\alpha}$. The main difficulty is with the second term. It is estimated by noting that $f \in B_{p q}^{\alpha-1}$ and $0<\alpha-1<1$ for $1<\alpha<2$. In this case

$$
\begin{aligned}
& \int(\| f(\cdot-\eta)-\left.f \|_{p}|\sin \langle\eta, h\rangle| /|\eta|^{\alpha}\right)^{q}|\eta|^{-n} d \eta \\
& \leqslant|h|^{q} \int_{|\eta|<|h|^{-1}}\left(\|f(\cdot-\eta)-f\|_{p} /|\eta|^{\alpha-1}\right)^{q}|\eta|^{-n} d \eta \\
& \quad+2^{q}\|f\|_{p}^{q} \int_{|\eta|>|h|^{-1}}|\eta|^{-\alpha q-n} d \eta \\
& \leqslant|h|^{q} \dot{B}_{p q}^{\alpha-1}(f)+2^{q}\|f\|_{p}^{q}|h|^{\alpha q} ;
\end{aligned}
$$

when $\alpha=1$, we use the fact that $f \in B_{p q}^{1} \subseteq B_{p q}^{1-\delta}$ for any $\delta>0$ and estimate the second term by

$$
\begin{aligned}
|h|^{\delta q} \int_{|\eta|<|h|^{-1}}(\| f(\cdot-\eta) & \left.-f \|_{p} /|\eta|^{1-\delta}\right)^{q}|\eta|^{-n} d \eta+2^{q}\|f\|_{p}^{q} \int_{|\eta|>|h|^{-1}}|\eta|^{-q-n} d \eta \\
& \leqslant|h|^{\delta q} \dot{B}_{p q}^{1-\delta}(f)+2^{q}\|f\|_{p}^{q}|h|^{q} .
\end{aligned}
$$

In this estimate we have taken $0<\delta<1$ and used the fact that for $0<x<$ $1, x^{\delta}>x$. This estimate finishes the proof in the case $0<\alpha<2$. Now it is easy to prove by induction that if $m<\alpha<m+1, f \in B_{p q}^{\alpha}$, then $e^{i\langle x, h\rangle} f \in$ $\dot{B}_{p q}^{\alpha}$ for every $h \in R^{n}$. The case $m=0$ has been proved above. If the result is true for $m$, consider an index $\alpha$ such that $m+1<\alpha<m+2$. Since $f \in$ $B_{p q}^{\alpha}$, the inhomogeneous Besov space, $f$ and $D_{j} f(j=1, \ldots, n)$ are in $B_{p q}^{\alpha-1}$, where $D_{j} f(x)=(1 / i) \partial f(x) / \partial x_{j}$. By the induction hypothesis, $f e^{i x h}$ and $\left(D_{j} f\right) e^{i\langle x, h\rangle}$ are in $\dot{B}_{p q}^{\alpha-1}$ for all $h$. Since 


$$
D_{j}\left(e^{i\langle x, h\rangle} f\right)=e^{i\langle x, h\rangle}\left(D_{j} f\right)+h_{j} e^{i x h} f,
$$

$D_{j}\left(e^{i\langle x, h\rangle} f\right) \in \dot{B}_{p q}^{\alpha-1}$ for $j=1,2, \ldots, n$, and it follows that $e^{i\langle x, h\rangle} f \in \dot{B}_{p q}^{\alpha}$.

The proof of the converse is more direct and can be given at one stroke. Since $\dot{B}_{p q}^{\alpha} \subseteq \dot{B}_{p \infty}^{\alpha}$ for any $q$, it is enough to prove that if $e^{i\langle x, h\rangle} f \in \dot{B}_{p \infty}^{\alpha}$ then $f \in L^{p}\left(R^{n}\right)$, since $B_{p q}^{\alpha}=\dot{B}_{p q}^{\alpha} \cap L^{p}$ for $\alpha>0$. Let $k$ be an integer $>\alpha$ and recall that $g$ belongs to $\dot{B}_{p \infty}^{\alpha}$ iff $\left\|\Delta_{\eta}^{k} g\right\|_{p}<A|\eta|^{\alpha}$. Recall that by definition

$$
\Delta_{\eta}^{k}\left(e^{i\langle x, h\rangle} f\right)=\sum_{0}^{k}\left(\begin{array}{l}
k \\
j
\end{array}\right)(-1)^{k-j} f(x+j \eta) e^{i\langle x+j, h\rangle},
$$

and multiplying by $e^{-i\langle x, h\rangle}$ gives

$$
e^{-i\langle x, h\rangle} \Delta_{\eta}^{k}\left(e^{i\langle x, h\rangle} f\right)=\sum_{0}^{k}\left(\begin{array}{l}
k \\
j
\end{array}\right)(-1)^{k-j} w_{h}^{j} f(x+j \eta),
$$

where $w_{h}=e^{i\langle\eta, h\rangle}$. Fix $\eta$ and choose $h_{0}, h_{1}, \ldots, h_{k}$ such that $w_{h_{i}}=w^{j}$, where $w^{k+1}=1$ but $w^{j} \neq 1$ for $j=1, \ldots, k$. Let $A_{m}=e^{-i\left\langle x, h_{m}\right\rangle} \Delta_{\eta}^{k}\left(e^{i\left\langle x, h_{m}\right\rangle} f\right)$, and an easy calculation gives $\Sigma_{0}^{k} A_{m}=(-1)^{k}(k+1) f(x)$, which allows us to make the estimate

$$
\|f\|_{p} \leqslant \frac{1}{k+1} \sum_{0}^{k}\left\|A_{m}\right\|_{p}<\frac{1}{k+1}\left[\sum_{0}^{k} \dot{B}_{p \infty}^{\alpha}\left(e^{\left.i<x, h_{m}\right\rangle} f\right)\right]|\eta|^{\alpha} .
$$

REMARK. Note that the choice of the $h_{j}$ depends on $\eta$, and if we make no assumption on the behavior of $\dot{B}_{p \infty o}^{\alpha}\left(e^{i\langle x, h\rangle} f\right)$ as a function of $h$, we cannot make the estimate of the $L^{p}$ norm of $f$ explicit. It seems that the possible estimate is of the nature of

$$
\dot{B}_{p q}^{\alpha}\left(e^{i<x, h\rangle} f\right)<C\left[\sum_{0<k<\alpha} \dot{B}_{p q}^{\alpha-k}(f)|h|^{k}+|h|^{\alpha}\|f\|_{p}\right] .
$$

With the behavior for $\alpha>0$ completely settled, we turn to the case $\alpha=0$. In this case the Littlewood-Paley properties of the homogeneous Besov spaces play a critical role.

THEOREM 1.2.

$$
\pi \dot{B}_{p \infty}^{0}\left(R^{n}\right)= \begin{cases}L^{p}\left(R^{n}\right), & 1<p<\infty, \\ \Re\left(R^{n}\right), & 1=p .\end{cases}
$$

Here $\mathscr{T}$ denotes the space of integrable measures on $R^{n}$.

Our approach is to derive an estimate of the $L^{p}$ norm of the temperature with initial value $e^{i x h} f$. For this, we need an estimate of an extension of the fundamental solution of the heat equation to complex $n$-space. It is clear that the result below can be generalized to estimate $W(x, s)=(4 \pi s)^{-n / 2} \exp (-x$. $x / 4 s), x \in C^{n}, \operatorname{Re} s>0$, but we do not need the result in this generality. 
LEMMA 1.1. Consider the function $f(x)=W(x+2 i s h, s), s>0$. Then, if $\alpha>-n / p^{\prime}, 1<q<\infty$, or if $\alpha>-n / p^{\prime}, q=\infty, f \in \dot{B}_{p q}^{\alpha}$ and

$$
\dot{B}_{p q}^{\alpha}(f)<A\left(1+\sqrt{s}|h|+\cdots+(\sqrt{s}|h|)^{3 k}\right) s^{-\alpha / 2-n / 2 p^{\prime}} e^{c s|h|^{2}},
$$

where $k$ is the smallest nonnegative integer greater than $\alpha / 2$, and $A, c$ are independent of $f$.

Proof. The temperature with initial value $f$ is

$$
u(x, t)=W(x, t+s) \exp (-i s\langle h, x\rangle / t+s) \exp \left(s^{2}|h|^{2} / t+s\right) .
$$

Consider first $\alpha<0$. The $\dot{B}_{p q}^{\alpha}$ norm of $f$ is $\left(\int_{0}^{\infty}\left[t^{-\alpha / 2} M_{p}(u ; t)\right]^{q} d t / t\right)^{1 / q}$, and an easy computation gives

$$
M_{p}(u ; t)=B_{p} \exp \left(s^{2}|h|^{2} / t+s\right)(s+t)^{-n / 2 p^{\prime}} .
$$

For $0 \leqslant t \leqslant 2 s, t^{-\alpha / 2} M_{p}(u ; t)$ is bounded above and below by $t^{-\alpha / 2} s^{-n / 2 p^{\prime}} \exp \left(c s|h|^{2}\right)$ and this gives a convergent $t$-integral for all $q$. For $t>2 s$, we can bound $t^{-\alpha / 2} M_{p}(u ; t)<C t^{-\alpha / 2-n / 2 p^{\prime}}$ and the right-hand side is in $L^{q}$ iff $\alpha>-n / p^{\prime}$ if $1<q<\infty$ or iff $\alpha>-n / p^{\prime}$ if $q=\infty$. While there is no corresponding lower bound, direct inspection shows that if $\alpha<-n / p^{\prime}$, $1<q<\infty$, or if $\alpha<-n / p^{\prime}, q=\infty$, the integral (or supremum) is infinite. In this case, the norm of $f$ is estimated by $\dot{B}_{p q}^{\alpha}(f)<A s^{-\alpha / 2-n / 2 p^{\prime}} \exp \left(s|h|^{2}\right)$.

For $\alpha>0$, let $k$ be a positive integer $>\alpha / 2$. An equivalent norm on $\dot{B}_{p q}^{\alpha}$ is given by

$$
\left(\int_{0}^{\infty}\left[t^{k-\alpha / 2} M_{p}\left(\partial_{t}^{k} u ; t\right)\right]^{q} t^{-1} d t\right)^{1 / q}
$$

The formula for $u$ was given above; apply Leibniz to it and it follows that

$$
\begin{aligned}
\partial_{t}^{k} u(x, t)= & \sum_{k_{1}+k_{2}+k_{3}=k} C_{k_{1} k_{2} k_{3}} \partial_{t}^{k_{1}} W(x, t+s) \partial_{t}^{k_{2}} \\
& \cdot \exp (-i\langle s, h\rangle / t+s) \partial_{t}^{k_{3}} \exp \left(s^{2}|h|^{2} / t+s\right) .
\end{aligned}
$$

One checks easily by induction that

$$
\begin{aligned}
\exp (+i s\langle h, x\rangle / t+s) \partial_{t}^{k} & (\exp (-i s\langle h, x\rangle / t+s)) \\
= & \sum_{j=1}^{k} C_{j}(s\langle h, x\rangle)^{j}(t+s)^{-k-j}
\end{aligned}
$$

and

$$
\exp \left(-s^{2}|h|^{2} / t+s\right) \partial_{t}^{k}\left(\exp \left(s^{2}|h|^{2} / t+s\right)\right)=\sum_{j=1}^{k} b_{j}\left(s^{2}|h|^{2}\right)^{j} /(t+s)^{-k-j}
$$

Substituting these expressions and the formula derived in [10] for $\partial_{t}^{k} W(x, t$ $+s)$, it is clear that $\partial_{t}{ }^{k} u$ is a finite sum of terms of the form 


$$
\begin{aligned}
\left(|x|^{2} / t+s\right)^{j_{1}} & (t+s)^{-k_{1}} W(x, t+s)\left(s^{2}|h|^{2}\right)^{j_{2}} \\
& \cdot(t+s)^{-k_{2}-j_{2}}(s\langle h, x\rangle)^{j_{3}}(t+s)^{-k_{3}-j_{3}} \\
\cdot & \exp (i s\langle h, x\rangle / t+s) \exp \left(s^{2}|h|^{2} / t+s\right),
\end{aligned}
$$

where $0<j_{1}<k_{1}, 1<j_{2}<k_{2}, 1<j_{3}<k_{3}$. The $L^{p}$ norm in $x$ of such an expression is bounded by

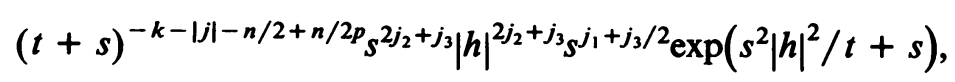

with $|j|=j_{1}+j_{2}+j_{3}$. Near the origin (in $t$-coordinates) such an expression is bounded by

$$
s^{-k-|j|-n / 2+n / 2 p+j_{1}+2 j_{2}+(3 / 2) j_{3}}|h|^{2 j_{2}+j_{3}} \exp \left(s|h|^{2}\right),
$$

so that multiplication by $t^{k-\alpha / 2}$ and integration with respect to $t^{-1} d t$ from say $t=0$ to $t=2 \mathrm{~s}$ gives a contribution of

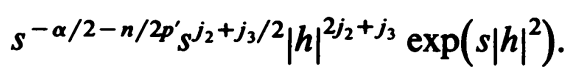

Near infinity $(t>2 s)$, the $L^{p}$ norm in $x$ is bounded by

$$
t^{-k-|j|-n / 2+n / 2 p^{\prime}}(s|h|)^{2 j_{2}+j_{3}} s^{j_{1}+j_{3} / 2} \exp \left(\frac{1}{3} s|h|^{2}\right),
$$

which after multiplication by $t^{k-\alpha / 2}$ and integration from $2 s$ to infinity gives a term of the form

$$
s^{-\alpha / 2-n / 2 p^{\prime}}\left(s|h|^{2}\right)^{j_{1}+j_{3} / 2}
$$

provided $-\alpha / 2-|j|-n / 2 p^{\prime}<0$, which in the worst case, is the requirement that $\alpha>-n / p^{\prime}$ (the term is bounded if $\alpha>-n / p^{\prime}$ ). Adding these two terms, we see that if $\alpha \geqslant 0, \alpha>-n / p^{\prime}(1<q<\infty)$ or $\alpha>-n / p^{\prime}$ $(q=\infty)$, then

$$
\dot{B}_{p q}^{\alpha}(f)<A s^{-\alpha / 2-n / 2 p^{\prime}} \exp \left(s|h|^{2}\right) \sum_{j=1}^{3 k / 2}\left(s|h|^{2}\right)^{j},
$$

where $j$ ranges over integers and integers $+1 / 2$ in the indicated range.

With this result, it is easy to give an estimate of the $L^{p}$ norm of the temperature with initial value $e^{i\langle x, h\rangle} f$.

LEMMA 1.2. If $e^{i\langle x, h\rangle} f \in \dot{B}_{p \infty}^{\alpha}\left(R^{n}\right), \alpha<1$, then

$$
\begin{aligned}
& \left(\int\left|W_{t} *\left(e^{i\langle x, h\rangle} f(x)\right)\right|^{p} d x\right)^{1 / p} \\
& \quad<A\left\{\dot{B}_{p \infty}^{\alpha}\left(e^{i\langle x, h\rangle} f\right)\left(\frac{1}{t|h|^{2}}+\frac{1}{\sqrt{t}|h|}\right)+\dot{B}_{p \infty}^{\alpha}(f) \sum_{j=-1}^{3 k / 2-1}\left(t|h|^{2}\right)^{j}\right\} t^{\alpha / 2} .
\end{aligned}
$$


Proof. Since $\alpha<2$, we must form $V_{h}(x, t)=W_{t} * e^{i\langle x, h\rangle} f$ and study $\partial V_{h} / \partial t$ in order to compute the $\dot{B}_{p \infty}^{\alpha}$ norm of $e^{i\langle x, h\rangle} f$. It is easily checked that $\partial V_{h} / \partial t=W_{t} * \Delta\left(e^{i\langle x, h\rangle} f\right)$ which can be rewritten as

$$
\begin{aligned}
\frac{\partial V_{h}}{\partial t} & =W_{t} *\left(|h|^{2} e^{i\langle x, h\rangle} f+2 i \sum_{j} h_{j} \frac{\partial}{\partial x_{j}}\left(f e^{i x h}\right)+e^{i x h} \Delta f\right) \\
& =|h|^{2} V_{h}(x, t)+2 i \sum_{j} h_{j} W_{t} * \frac{\partial}{\partial x_{j}}\left(f e^{i\langle x, h\rangle}\right)+W_{t} * e^{i x h} \Delta f,
\end{aligned}
$$

which allows us to express $V_{h}$ in terms of $\partial V_{h} / \partial t$ by

$$
|h|^{2} V_{h}(x, t)=\frac{\partial V_{h}}{\partial t}-2 i \sum_{j=1}^{n} h_{j} W_{t} * \frac{\partial}{\partial x_{j}}\left(f e^{i x h}\right)-W_{t} * e^{i x h} \Delta f
$$

We must now compute the norm of each of the terms on the right-hand side in terms of the $\dot{B}_{p \infty}^{\alpha}$ norm. The first term is straightforward and

$$
M_{p}\left(\partial V_{h} / \partial t, t\right)<\dot{B}_{p \infty}^{\alpha}\left(e^{i\langle x, h\rangle} f\right) t^{\alpha / 2-1},
$$

and since $\left(\partial / \partial x_{j}\right)\left(e^{i\langle x, h\rangle} f\right) \in \dot{B}_{p \infty}^{\alpha-1}$ and $\alpha-1<0$,

$$
M_{p}\left(W_{t} *\left(\partial / \partial x_{j}\right)\left(f e^{i\langle x, h\rangle}\right)\right)<\dot{B}_{p \infty}^{\alpha}\left(f e^{i x h}\right) t^{(\alpha-1) / 2} .
$$

Lemma 1.1 can be applied to the third term if we rewrite it as

$$
w_{h}(x, t)=W_{t} * e^{i\langle x, h\rangle} \Delta f=e^{-i|h|^{2}} e^{i\langle x, h\rangle} g * \Delta f,
$$

where $g(x)=W(x+2 i t h, t)$. Observe that $\Delta f \in \dot{B}_{p \infty}^{\alpha-2}$ and that for any $p$, $\dot{B}_{p 1}^{0} \subset L^{p}\left(R^{n}\right)$, and we get the estimate

$$
\begin{aligned}
M_{p}\left(w_{h} ; t\right) & <e^{-t|h|^{2}}\|g * \Delta f\|_{p}<A e^{-t|h|^{2}}\|g * \Delta f\|_{\dot{B}_{p 1}^{0}} \\
& <A e^{-t|h|^{2}}\|g\|_{\dot{B}_{11}^{2}-\alpha}\|\Delta f\|_{\dot{B}_{p \infty}^{\alpha-2 .}}
\end{aligned}
$$

Since $\alpha<1,2-\alpha>1$ and when we apply Lemma 1.1 there results

$$
M_{p}\left(w_{h} ; t\right)<A e^{-t|h|^{2}} e^{t|h|^{2}} t^{-((2-\alpha) / 2)}\left(\sum_{0}^{k}\left(t|h|^{2}\right)^{j}\right) \dot{B}_{p \infty}^{\alpha}(f) .
$$

Collecting these three terms together, we have

$$
\begin{aligned}
|h|^{2} M_{p}\left(V_{h} ; t\right)< & A \dot{B}_{p \infty}^{\alpha}\left(e^{i\langle x, h\rangle} f\right)\left[t^{\alpha / 2-1}+|h| t^{(\alpha-1) / 2}\right] \\
& +A \dot{B}_{p \infty}^{\alpha}(f) t^{\alpha / 2-1} \sum_{0}^{k}\left(t|h|^{2}\right)^{j}
\end{aligned}
$$


or finally,

$$
\begin{aligned}
M_{p}\left(V_{h} ; t\right)<A t^{\alpha / 2}\left\{\dot{B}_{p \infty}^{\alpha}\left(e^{i\langle x, h\rangle} f\right)\right. & {\left[\frac{1}{\sqrt{t}|h|}+\frac{1}{t|h|^{2}}\right] } \\
& \left.+\dot{B}_{p \infty}^{\alpha}(f) \frac{1}{t|h|^{2}}\left(\sum_{0}^{k}\left(t|h|^{2}\right)^{j}\right)\right\}
\end{aligned}
$$

This concludes the proof of Lemma 1.2.

We would now like to let $t \rightarrow 0+$, but the right-hand side is not suitably behaved; we will need to make a judicious choice of $h=h(t)$.

Proof of THEOREM 1.2. Suppose $e^{i x h} f \in \dot{B}_{p \infty}^{0}$ for each $h \in R^{n}$ and $\dot{B}_{p \infty}^{0}\left(e^{i\langle x, h\rangle} f\right)<A \dot{B}_{p \infty}^{0}(f)$. Lemma 1.2 gives in this case

$$
\left\|W_{t} *\left(e^{i\langle x, h\rangle} f\right)\right\|_{p}<A \dot{B}_{p \infty}^{0}(f)\left[\frac{1}{t|h|^{2}}+\frac{1}{\sqrt{t}|h|}+1+\sqrt{t}|h|+t|h|^{2}\right] .
$$

In particular, we can apply this estimate to $e^{i\langle x, \eta\rangle} f$, which gives

$$
\begin{aligned}
\left\|W_{t} * e^{i\langle x, \xi\rangle} f\right\|_{p} & \leqslant A \dot{B}_{p \infty}^{0}\left(e^{i\langle x, \eta\rangle} f\right)\left[\frac{1}{t|h|^{2}}+\cdots+t|h|^{2}\right] \\
& <A_{1} \dot{B}_{p \infty}^{0}(f)\left[\frac{1}{t|h|^{2}}+\cdots+t|h|^{2}\right],
\end{aligned}
$$

where $\xi=h+\eta$. Choose $h=h(t)$ such that $t|h|^{2}=1$, and since everything is independent of $\eta$, choose $\eta(t)=-h(t)$. We see that

$$
\left\|W_{t} * f\right\|_{p} \leqslant A^{\prime \prime} \dot{B}_{p \infty}^{0}(f) \text {. }
$$

Now well-known results [8] imply the conclusion of the theorem.

The above proof is somewhat technical; we give another simpler proof of some special cases, which is based on a result of interest in its own right.

LEMMA 1.3. $\pi \mathrm{BMO}=L^{\infty}$

Proof. Since $L^{\infty} \subset$ BMO and $L^{\infty}$ is invariant under multiplication by characters, one direction is obvious. For the converse it will be shown that if $e^{i\langle x, h\rangle} f$ is of bounded mean oscillation for all $h$, the constant in the definition of BMO can always be taken to be zero and thus $f \in L^{\infty}$.

Let $Q$ be a cube in $R^{n}$, and $f, g$ be arbitrary functions of bounded mean oscillation. Denote

$$
f_{Q}=\frac{1}{|Q|} \int_{Q} f(x) d x, \quad\|f\|_{*}=\sup _{Q}\left(\frac{1}{|Q|} \int_{Q}\left|f(x)-f_{Q}\right|^{2} d x\right)^{1 / 2}
$$


and observe that

$$
\begin{aligned}
(f g)_{Q}-f_{Q} g_{Q} & =\frac{1}{|Q|} \int_{Q}\left[f(x)-f_{Q}\right] g(x) d x \\
& =\frac{1}{|Q|} \int_{Q}\left(f(x)-f_{Q}\right)\left(g(x)-g_{Q}\right) d x
\end{aligned}
$$

which gives by Hölder's inequality $\left|(f g)_{Q}-f_{Q} g_{Q}\right|<\|f\|_{*}\|g\|_{*}$. If we apply this inequality to $e^{i\langle x, h\rangle} f$, it is clear that

$$
\begin{aligned}
\frac{1}{|Q|} \int_{Q}\left|e^{i\langle x, h\rangle} f(x)-\left(e^{i\langle x, h\rangle}\right) f_{Q}\right| d x \\
\quad<\frac{1}{|Q|} \int_{Q}\left|\left(e^{i\langle x, h\rangle} f\right)(x)-\left(e^{i\langle x, h\rangle} f\right)_{Q}\right| d x+\left|\left(e^{i\langle x, h\rangle} f\right)_{Q}-\left(e^{i\langle x, h\rangle}\right)_{Q} f_{Q}\right| \\
<\left\|e^{i\langle x, h\rangle} f\right\|_{*}+\left\|e^{i\langle x, h\rangle}\right\|_{*}\|f\|_{*},
\end{aligned}
$$

and if $\left\|e^{i\langle x, h\rangle} f\right\|_{*}<A\|f\|_{*}$, we see that

$$
\frac{1}{|Q|} \int_{Q}\left|e^{i\langle x, h\rangle} f(x)-\left(e^{i\langle x, h\rangle}\right)_{Q} f_{Q}\right| d x<A^{\prime}\|f\|_{*}
$$

Choose $h(Q)$ such that $\left(e^{i\langle x, h\rangle}\right)_{Q}=0$, and then

$$
\frac{1}{|Q|} \int_{Q}|f(x)| d x<A^{\prime}\|f\|_{*}
$$

Since $A^{\prime}$ is independent of $Q$, Lebesgue's theorem on differentiation of the integral gives the result.

This lemma allows us to prove the next result which includes Theorem 1.2 in some special cases.

THEOREM 1.3.

$$
\pi C v\left(H^{1}, L^{p}\right)= \begin{cases}L^{p}\left(R^{n}\right), & 1<p<\infty \\ \Re\left(R^{n}\right), & p=1\end{cases}
$$

It is known [9] that $C v\left(H^{1}, L^{p}\right)=\dot{B}_{p \infty}^{0}$ when $2<p<\infty$; in the case $1<p<2$, there is an inclusion but the above result applied to the inclusion gives no new results.

Proof OF THeOREM 1.3. One direction is obvious. Suppose that $e^{i\langle x, h\rangle} k \in$ $C v\left(H^{1}, L^{p}\right)$ for every $h \in R^{n}$ and that its norm as a convoluteur is bounded independently of $h$. Note first that a distribution $T$ which maps $H^{1} \rightarrow L^{p}$ boundedly will also map $L^{p^{\prime}} \rightarrow$ BMO and satisfy $\|T(g)\|_{\text {BMO }}<A\|T\|\|g\|_{p^{\prime}}$ Fix $g \in L^{p^{\prime}}$. From this remark, we know that $k * g \in \mathrm{BMO}$; we will show that $e^{i\langle x, h\rangle}(k * g)$ is also in BMO. Indeed,

$$
e^{i\langle x, h\rangle} k * g(x)=\left\langle e^{i\langle y, h\rangle} k_{y}, e^{i\langle x-y, h\rangle} g(x-y)\right\rangle \text {, }
$$


and thus $e^{i\langle x, h\rangle} k * g \in \mathrm{BMO}$ and

$$
\left\|e^{i\langle x, h\rangle}(k * g)\right\|_{\mathrm{BMO}} \leqslant A\left\|e^{i\langle\cdot h\rangle} k\right\|\left\|e^{i\langle\cdot, h\rangle} g\right\|_{p^{\prime}} \leqslant A^{\prime}\|g\|_{p^{\prime}}\|k\| .
$$

By Lemma 1.3, we see that $k * g \in L^{\infty}\left(R^{n}\right)$ and $\|k * g\|_{\infty}<A^{\prime \prime}\|k\|\|g\|_{p^{\prime}}$, which proves that $k \in C v\left(L^{p^{\prime}}\left(R^{n}\right), L^{\infty}\left(R^{n}\right)\right)=C v\left(L^{1}\left(R^{n}\right), L^{p}\left(R^{n}\right)\right)$ which is the result.

Another interesting consequence of Lemma 1.3 is the following characterization of $L^{\infty}$ in terms of $L^{2}$ properties of the temperature associated with a bounded function.

Proposition 1. Let $f$ be a distribution in $\mathcal{S}^{\prime}$ and $u$ be the temperature associated with $f$. Then $f \in L^{\infty}$ iff for every $h \in R^{n}, \delta>0, x_{0} \in R^{n}$,

$$
\int_{0}^{\delta} \int_{\left|x-x_{0}\right|<\delta} t\left|\nabla\left(e^{i\langle x, h\rangle} e^{-t|h|^{2}} u(x+2 i t h, t)\right)\right|^{2} d t d x<C \delta^{n}
$$

Proof. This follows from Lemma 1.3, the fact that the temperature with initial value $e^{i\langle x, h\rangle} f$ is $e^{i\langle x, h\rangle} e^{-t|h|^{2}} u(x+2 i t h, t)$ and the characterization of BMO in [3].

The next result, properly speaking, belongs in $\$ 2$ but is included here because it concludes the (short) list of Besov spaces for which we can characterize $\pi \dot{B}_{p \infty}^{\alpha}$. The methods are based on the Fourier transform and the use of the $K_{p q}^{\alpha}$ spaces. We need two auxiliary results.

LEMMA 1.4. $K_{p \infty}^{\alpha} \subseteq L_{\text {loc }}^{1}$, if $\alpha<n / p^{\prime}$.

Proof. An equivalent norm on $K_{p \infty}^{\alpha}$ is provided by $\sup _{t} t^{-(\beta-\alpha)}\left(\int_{|x|<t}|x|^{p \beta}|f(x)|^{p} d x\right)^{1 / p}$ for $\beta>\alpha$ [5]. If $\alpha<n / p^{\prime}$, choose $\alpha<$ $\beta<n / p^{\prime}$ and estimate

$$
\begin{aligned}
\int_{|x|<t}|f(x)| d x & \leqslant\left(\int_{|x|<t}\left(|f(x)||x|^{\beta}\right)^{p} d x\right)^{1 / p}\left(\int_{|x|<t}|x|^{-\beta p^{\prime}} d x\right)^{1 / p^{\prime}} \\
& \leqslant K_{p \infty}^{\alpha}(f) t^{\beta-\alpha}\left(w_{n} \int_{0}^{t} \rho^{-\beta p^{\prime}+n} \rho^{-1} d \rho\right)^{1 / p^{\prime}}
\end{aligned}
$$

and this last integral is finite since $n-\beta p^{\prime}>0$.

LEMMA 1.5. (a) If $f \in \dot{B}_{p \infty}^{\alpha}, 1 \leqslant p<2, \alpha<-n / p^{\prime}$, then for any $R>0$,

$$
\left(\int_{|\xi|<R}|\hat{f}(\xi)|^{p^{\prime}} d \xi\right)^{1 / p^{\prime}}<A R^{-\alpha} \dot{B}_{p \infty}^{\alpha}(f),
$$

(b) If $\in \dot{B}_{p q}^{-n / p^{\prime}}, 1 \leqslant p \leqslant 2,1 \leqslant q<\infty$, then

$$
\left[\int_{0}^{\infty}\left(s^{-n} \int_{|\xi|<s}|\hat{f}(\xi)|^{p^{\prime}} d \xi\right)^{q / p^{\prime}} s^{-1} d s\right]^{1 / q} \leqslant A \dot{B}_{p q}^{-n / p^{\prime}}(f) .
$$


Proof. (a) Since $\alpha<0, u(x, t)=f * W_{t}$ satisfies $t^{-\alpha / 2} M_{p}(u ; t)<$ $A \dot{B}_{p \infty}^{\alpha}(f)$, and by Hausdorff-Young we get $t^{-\alpha / 2} M_{p^{\prime}}(\hat{u} ; t)<A \dot{B}_{p \infty}^{\alpha}(f)$. The left-hand side can be estimated from below by

$$
\begin{aligned}
M_{p^{\prime}}(\hat{u} ; t) & =\left(\int\left|e^{-t|\xi|^{2}} \hat{f}(\xi)\right|^{p^{\prime}} d \xi\right)^{1 / p^{\prime}}>\left(\int_{|\xi|<1 / \sqrt{t}}\left|e^{-t|\xi|^{2}} \hat{f}(\xi)\right|^{p^{\prime}} d \xi\right)^{1 / p^{\prime}} \\
& \geqslant e^{-1}\left(\int_{|\xi|<1 / \sqrt{t}}|\hat{f}(\xi)|^{p^{\prime}} d \xi\right)^{1 / p^{\prime}} .
\end{aligned}
$$

Let $R=1 / \sqrt{t}$ and we can rewrite this as

$$
\left(\int_{|\xi|<R}|\hat{f}(\xi)|^{p^{\prime}} d \xi\right)^{1 / p^{\prime}}<e M_{p^{\prime}}(\hat{u} ; t)<A \dot{B}_{p \infty}^{\alpha}(f) R^{-\alpha} .
$$

(b) The argument is as above, but we begin with the fact that

$$
\left(\int_{0}^{\infty}\left[t^{-\alpha / 2} M_{p}(u ; t)\right]^{q} t^{-1} d t\right)^{1 / q}=\dot{B}_{p q}^{\alpha}(f)
$$

and proceed to

$$
\begin{gathered}
\left(\int_{0}^{\infty}\left[t^{-\alpha / 2} M_{p^{\prime}}(\hat{u} ; t)\right]^{q} t^{-1} d t\right)^{1 / q}<\dot{B}_{p q}^{\alpha}(f) \\
{\left[\int_{0}^{\infty}\left[t^{-\alpha / 2}\left(\int_{|\xi|<1 / \sqrt{t}}|\hat{f}(\xi)|^{p^{\prime}} d \xi\right)^{1 / p^{\prime}}\right]^{q} t^{-1} d t\right]^{1 / q}<e B_{p q}^{\alpha}(f) .}
\end{gathered}
$$

A change of variables $t \rightarrow s=1 / \sqrt{t}$ gives the result.

There are two applications of Lemma 1.5, one local and one global. The global application is to the determination of $\pi \dot{B}_{p \infty}^{\alpha}$.

THEOREM 1.4. $\pi \dot{B}_{p q}^{\alpha}\left(R^{n}\right)=(0)$, if $\alpha<-n / p^{\prime}$ or if $\alpha<-n / p^{\prime}, 1<q<$ $\infty$, for $1<p<2$.

Proof. For $\alpha<-n / p^{\prime}$, we may assume that $q=\infty$ since $\dot{B}_{p q}^{\alpha} \subset \dot{B}_{p \infty}^{\alpha}$. If $f \in \pi \dot{B}_{p q}^{\alpha}$, we see from Lemma 1.5 that

$$
\left(\int_{|\xi|<R}|\hat{f}(\xi+h)|^{p^{\prime}} d \xi\right)^{1 / p^{\prime}}<A \dot{B}_{p \infty}^{\alpha}\left(e^{-i\langle x, h\rangle} f\right) R^{-\alpha}<A \dot{B}_{p \infty}^{\alpha}(f) R^{-\alpha} .
$$

Raise this inequality to the $p^{\prime}$ th power $(1<p<2)$ and change variables and we have

$$
\frac{1}{b_{n} R^{n}} \int_{|\xi-h|<R}|\hat{f}(\xi)|^{p^{\prime}} d \xi<A^{p^{\prime} \dot{B}_{p \infty}^{\alpha}}(f)^{p^{\prime}} R^{-\alpha p^{\prime}-n}
$$


Note that $\hat{f} \in K_{p^{\prime} \infty}^{\alpha} \subseteq L_{\text {loc }}^{1}$ by Lemma 1.4, and hence almost every point of $R^{n}$ is a Lebesgue point of $\hat{f}$. Letting $R \rightarrow 0+$ at the Lebesgue points gives $\hat{f}(h)=0$ a.e., or $f=0$ as a distribution. (If $p=1$, the result is trivial.)

For $1<q<\infty$ and $f \in \dot{B}_{p^{\prime} \infty}^{-n / p^{\prime}}$, Lemma 1.5 gives

$$
\left.\iint_{0}^{\infty}\left(\frac{1}{b_{n} s^{n}} \int_{|\xi-h|<s}|\hat{f}(\xi)|^{p^{\prime}} d \xi\right)^{q / p^{\prime}} s^{-1} d s\right]^{1 / q}<A \dot{B}_{p q}^{-n / p^{\prime}}(f) .
$$

At a Lebesgue point of $\hat{f}$ (one can again check that $\hat{f}$ is locally integrable), the inner integral can converge only if $\hat{f}(h)=0$ so we again conclude that $f=0$ as a distribution.

The lemma can also be applied locally in $h$, and then gives a sufficient condition for a distribution $f$ to have a spectral gap.

Corollary 1.5.1. Suppose that for $|h-a|<\delta_{0}, e^{-i\langle x, h\rangle} f \in \dot{B}_{p q}^{\alpha}, 1<p<$ 2, $\alpha<-n / p^{\prime}$ if $q=\infty$ or $\alpha<-n / p^{\prime}$ if $1<q<\infty$ and $\dot{B}_{p q}^{\alpha}\left(e^{-i\langle x, h\rangle} f\right)<$ $A \dot{B}_{p q}^{\alpha}(f)$ for $|h-a|<\delta_{0}$. Then $f$ has a spectral gap at a.

Unfortunately the analogue of Theorem 1.4 is definitely false for some $p>2$. For example $\dot{B}_{\infty \infty}^{\alpha}\left(R^{n}\right)$ contains the subalgebra $\left\{e^{i\langle x, h\rangle} \mid h \in R^{n}\right\}$ for any real $\alpha$.

There are various applications of these results which will be collected in $\$ 3$.

2. Translation invariant subspaces of $K_{p q}^{\alpha}$. The main gap that remains in our results is for indices $\alpha$ such that $-n / p^{\prime}<\alpha<0$ and $\alpha=0,1<q<\infty$. Some indirect information can be obtained by taking Fourier transforms and studying the translation invariant subspaces of $K_{p q}^{\alpha}$.

Our first result is an inequality giving a lower bound for the norm of the translates. This inequality is sharp enough to solve our problem in several cases.

THEOREM 2.1. Suppose that $\tau_{h} f, \tau_{-h} f$ are in $K_{p q}^{\alpha}$ for some measurable function $f$, where $\alpha$ is real and $1<p, q<\infty$. It follows that, for any $\beta>\alpha, \beta>0$,

$$
\begin{array}{r}
|h|^{\beta}\left(\int_{|h|}^{\infty}\left(t^{-p(\beta-\alpha)} \int_{|z|<t}|f(z)|^{p} d z\right)^{q / p} t^{-1} d t\right)^{1 / q} \\
<A\left(K_{p q}^{\alpha}\left(\tau_{h} f\right)+K_{p q}^{\alpha}\left(\tau_{-h} f\right)\right) .
\end{array}
$$

Here $A$ is independent of $f$ and $h$ but depends on all the other parameters. The obvious interpretations are to be made when $p=\infty$ or $q=\infty, e . g$.

$$
\sup _{t>|h|} t^{-(\beta-\alpha)}|h|^{\beta}\left(\int_{|z|<t}|f(z)|^{p} d z\right)^{1 / p}<A\left(K_{p \infty}^{\alpha}\left(\tau_{h} f\right)+K_{p \infty}^{\alpha}\left(\tau_{-h} f\right)\right) .
$$



by

Proof. If $\beta>\alpha$, it is proved in [5] that an equivalent norm on $K_{p q}^{\alpha}$ is given

$$
K_{p q}^{\alpha}(f)=\left(\int_{0}^{\infty}\left(t^{-p(\beta-\alpha)} \int_{|x|<t}|x|^{p \beta}|f(x)|^{p} d x\right)^{q / p} t^{-1} d t\right)^{1 / q},
$$

and we apply this norm to $\tau_{h} f$ and change variables. This gives

$$
\left.\iint_{0}^{\infty}\left(t^{-p(\beta-\alpha)} \int_{|z+h|<t}|z+h|^{p \beta}|f(z)|^{p} d z\right)^{q / p} t^{-1} d t\right)^{1 / q} .
$$

For the moment we are assuming that $p, q\langle\infty$. If $\langle z, h\rangle\rangle 0$, it is easy to see that $|z+h| \tilde{\mid}|+| h \mid$ (in fact, $(1 / \sqrt{2})(|z|+|h|)<|z+h|<\sqrt{2}(|z|+|h|)$ ). If the integration is taken only over this smaller set, it follows that

$$
\int_{0}^{\infty}\left[t^{-p(\beta-\alpha)} \int_{\substack{|z+h|<t \\\langle z, h\rangle>0}}(|z|+|h|)^{p \beta}|f(z)|^{p} d z\right)^{q / p} t^{-1} d t<A^{\prime} K_{p q}^{\alpha}\left(\tau_{h} f\right)^{q} .
$$

This is also valid for $-h$ which gives

$$
\int_{0}^{\infty}\left[t^{-p(\beta-\alpha)} \int_{\substack{|z-h|<t \\\langle z, h\rangle<0}}(|z|+|h|)^{p \beta}|f(z)|^{p} d z\right)^{q / p} t^{-1} d t<A^{\prime} K_{p q}^{\alpha}\left(\tau_{-h} f\right)^{q} .
$$

Next we observe that if $E_{1 t}=\left\{z|| z+h \mid\langle t,\langle z, h\rangle>0\}, E_{2 t}=\{t|| z-h \mid\right.$ $\langle t,\langle z, h\rangle<0\}$; then if $|h|<t / 2,\{z|| z \mid<t / 2\} \subseteq E_{1 t} \cup E_{2 t}$, and hence one sees that

$$
\begin{gathered}
\int_{2|h|}^{\infty}\left(t^{-p(\beta-\alpha)} \int_{|z|<t / 2}(|z|+|h|)^{p \beta}|f(z)|^{p} d z\right)^{q / p} t^{-1} d t \\
<A^{\prime}\left(K_{p q}^{\alpha}\left(\tau_{h} f\right)^{q}+K_{p q}^{\alpha}\left(\tau_{-h} f\right)^{q}\right) .
\end{gathered}
$$

A final change of variables in $t$ gives the result.

It is easily checked that only trivial changes need be make to get the result if $p$ or $q$ is infinity.

This result is first applied to the special case $p=q$.

THEOREM 2.2. If $\alpha$ is nonnegative and $1<p<\infty$. Then $\tau_{h} f \in K_{p p}^{\alpha}$ for every $h \in R^{n}$ iff $f \in L^{p} \cap K_{p p}^{\alpha}$, and one has

$$
\begin{aligned}
\varlimsup_{|h| \rightarrow \infty}\left[K_{p p}^{\alpha}\left(\tau_{h} f\right)+K_{p p}^{\alpha}\left(\tau_{-h} f\right)\right] /|h|^{\alpha} & \approx\|f\|_{p}, \\
\varlimsup_{|h| \rightarrow 0}\left[K_{p p}^{\alpha}\left(\tau_{h} f\right)+K_{p p}^{\alpha}\left(\tau_{-h} f\right)\right] & \approx K_{p p}^{\alpha}(f) .
\end{aligned}
$$


Proof. One direction is immediate if we note that if $f \in L^{p} \cap K_{p p}^{\alpha}$, then, if $\alpha>0$,

$$
\begin{aligned}
K_{p p}^{\alpha}\left(\tau_{h} f\right) & =\left(\int|f(x-h)|^{p} v(x)^{p \alpha / n} d x\right)^{1 / p} \\
& <2^{\alpha} b_{n}^{\alpha / n}\left(\int|f(z)|^{p}\left(|z|^{p \alpha}+|h|^{p \alpha}\right) d z\right)^{1 / p} \\
& <2^{\alpha} K_{p p}^{\alpha}(f)+2^{\alpha}|h|^{\alpha}\|f\|_{p} .
\end{aligned}
$$

Thus, we obtain

$$
K_{p p}^{\alpha}\left(\tau_{h} f\right)+K_{p p}^{\alpha}\left(\tau_{-h} f\right)<2^{\alpha+1} K_{p p}^{\alpha}(f)+2^{\alpha+1}|h|^{\alpha}\|f\|_{p},
$$

and the upper bounds follow.

Conversely, for any $\alpha$ real, if $\tau_{h} f \in K_{p p}^{\alpha}$ for all $h \in R^{n}$, we apply Theorem 2.1 and deduce that for any $\beta>\alpha$ with $\beta>0$, $|h|^{p \beta}\left(\int_{|h|}^{\infty}\left[t^{-p(\beta-\alpha)} \int_{|z|<t}|f(z)|^{p} d z\right] t^{-1} d t\right)<A^{p}\left[K_{p p}^{\alpha}\left(\tau_{h} f\right)^{p}+K_{p p}^{\alpha}\left(\tau_{-h} f\right)^{p}\right]$, and the left-hand side is

$$
\begin{aligned}
& |h|^{\beta p} \int|f(z)|^{p}\left(\int_{|z|<t,|h|<t} t^{-p(\beta-\alpha)} t^{-1} d t\right) d z \\
& \quad=|h|^{\beta p} \int_{|z|<|h|}|f(z)|^{p}|h|^{-p(\beta-\alpha)} d z+|h|^{\beta p} \int_{|z|>|h|}|f(z)|^{p}|z|^{-p(\beta-\alpha)} d z .
\end{aligned}
$$

We see then that

$$
\int_{|z|<|h|}|f(z)|^{p} d z<A^{p}\left[K_{p p}^{\alpha}\left(\tau_{h} f\right)^{p}+K_{p p}^{\alpha}\left(\tau_{-h} f\right)^{p}\right] /|h|^{p \alpha},
$$

and taking the $p$ th roots and the limit inferior as $|h| \rightarrow \infty$ gives the estimate

$$
\|f\|_{p}<A \lim _{|h| \rightarrow \infty} \frac{K_{p p}^{\alpha}\left(\tau_{h} f\right)+K_{p p}^{\alpha}\left(\tau_{-h} f\right)}{|h|^{\alpha}}
$$

The corresponding inequality for the $K_{p p}^{\alpha}$ norm of $f$ follows by direct substitution.

In the next result, we characterize those $f$ for which the translates behave in an expected manner in the range for which we were not able to give the corresponding results for Besov spaces.

THEOREM 2.3. A function $f$ has the property that $\tau_{h} f \in K_{p \infty}^{\alpha}$ for all $h \in R^{n}$ with norms bounded independently of the translate:
(a) if $\alpha=0$, iff $f \in L^{p}$ and $\|f\|_{p} \sim \sup _{h} K_{p \infty}^{0}\left(\tau_{h} f\right)$,
(b) if $-n / p<\alpha<0$, iff $f \in L^{p,-\alpha p}$ and $\|f\| \sim \sup _{h} K_{p \infty}^{\alpha}\left(\tau_{h} f\right)$,
(c) if $\alpha=-n / p$, iff $f \in L^{\infty}$ and $\|f\|_{\infty} \sim \sup _{h} K_{p \infty}^{-n / p}\left(\tau_{h} f\right)$,
(d) if $\alpha<-n / p$, iff $f \equiv 0$. 
For $\alpha>0$, our assumption would imply that $f \equiv 0$ also, but this is because we are not being flexible enough in the form of the dependence on $h$. Property (b) indicates (and (d) confirms) that asking this form of translation invariance is a stiff requirement. Property (c) gives an unexpected link with the Morrey spaces, which although valid for $-n / p<\alpha<0$, does not hold for $\alpha<-n / p$ since $L^{p, n}=\mathrm{BMO} \neq L^{\infty}$. It will follow from the proof below that $f \in L^{p,-\alpha p}$ in all cases and that the constant which occurs in the definition of the $L^{p, \lambda}$ spaces can always be taken equal to zero, which is known to be the case for $-n / p<\alpha<0$. Moreover, it is easy to see from the proof that (d) remains valid for $\alpha=-n / p, 1<q<\infty$.

Proof. We can write the conditions of Theorem 2.1 in the form

$$
|h|^{\beta} t^{-(\beta-\alpha)}\left(\int_{|z|<t}|f(z)|^{p} d z\right)^{1 / p} \leqslant A\left(K_{p \infty}^{\alpha}\left(\tau_{h} f\right)+K_{p \infty}^{\alpha}\left(\tau_{-h} f\right)\right),
$$

for $t \geqslant|h|$. We will take $\beta=\alpha+n / p$ and use only the result for $t=|h|$. For $\alpha=0$, we get

$$
\left(\int_{|z|<|h|}|f(z)|^{p} d z\right)^{1 / p} \leqslant A\left(K_{p \infty}^{\alpha}\left(\tau_{h} f\right)+K_{p \infty}^{\alpha}\left(\tau_{-h} f\right)\right),
$$

with $h$ arbitrary, which shows that $\|f\|_{\rho} \leqslant A \sup _{h} K_{p \infty}^{0}\left(\tau_{h} f\right)$. For $\alpha=-n / p$, we get

$$
\left(1 /|h|^{n} \int_{|z|<|h|}|f(z)|^{p} d z\right)^{1 / p}<A \sup _{h} K_{p \infty}^{-n / p}\left(\tau_{h} f\right),
$$

and since this will also be valid for $\tau_{y} f$, it follows from Lebesgue's theorem on the differentiation of integrals that $f \in L^{\infty}$ and $\|f\|_{\infty}<\sup _{h} K_{p}^{-n / p}\left(\tau_{h} f\right)$. If $-n / p<\alpha<0$, we have

$$
\left(\int_{|z|<R}|f(z)|^{p} d z\right)^{1 / p}<A\left[\sup _{h} K_{p \infty}^{\alpha}\left(\tau_{h} f\right)\right] R^{-\alpha},
$$

and since this holds for $\tau_{y} f$, we see that $f \in L^{p,-p \alpha}$.

In each case the converse is easily checked.

A particular corollary of this result is a complete characterization of $\pi \dot{B}_{2 \infty}^{\alpha}$ for $p=2, q=\infty$.

Corollary 2.1.1.

(a)

$$
\begin{aligned}
\left\{f \in \mathcal{S}^{\prime} \mid e^{i\langle x, h\rangle} f \in \dot{B}_{2 \infty}^{\alpha} \text { with } \dot{B}_{2 \infty}^{\alpha}\left(e^{i\langle x, h\rangle} f\right)<A_{1}(f)+A_{2}(f)|h|^{\alpha}, \forall h\right\} \\
=B_{2 \infty}^{\alpha}\left(R^{n}\right), \quad \alpha>0,
\end{aligned}
$$


(b)

$$
\begin{aligned}
\left\{f \in \mathcal{S}^{\prime} \mid e^{i\langle x, h\rangle} f \in \dot{B}_{2 \infty}^{\alpha} \text { with } \dot{B}_{2 \infty}^{\alpha}\left(e^{i\langle x, h\rangle} f\right)<A \dot{B}_{2 \infty}^{\alpha}(f), \forall h\right\} \\
= \begin{cases}L^{2}\left(R^{n}\right), & \alpha=0, \\
F L^{2,-2 \alpha}, & -n / 2<\alpha<0, \\
F L^{\infty}, & \alpha=-n / 2, \\
\{0\}, & \alpha<-n / 2 .\end{cases}
\end{aligned}
$$

3. Applications. Our first application is the one that motivated this paper. The first result was proved in [9].

THEOREM 3.1. $C v\left(L^{p}, L^{q}\right) \subseteq \dot{B}_{q \infty}^{n(1 / p-1)}$, if $p<q^{\prime}$.

For $p>q^{\prime}$, there is a better result. It now follows from Theorem 2.3 that

THEOREM 3.2.

$$
M\left(L^{p}, L^{q}\right) \subseteq \begin{cases}L^{\infty}, & p=q<2 \\ L^{q^{\prime}, n(p-1)}, & 1<p<q<2\end{cases}
$$

Proof. It follows from Theorem 3.1 that since $q<2, M\left(L^{p}, L^{q}\right) \subseteq$ $K_{q^{\prime} \infty}^{n(1 / p-1)}$. Theorem 1.13 of [7] implies that $M\left(L^{p}, L^{q}\right)$ is invariant under translations, which yields $M\left(L^{p}, L^{q}\right) \subseteq \tau K_{q^{\prime} \infty}^{n(1 / p-1)}$. The result follows from Theorem 2.3 if we note that when $p<q,-n / q^{\prime}<n(1 / p-1)<0$.

COROllary 3.2.1. $C v\left(L^{2}, L^{2}\right)$ is the maximal subspace of $\dot{B}_{2 \infty}^{-n / 2}$ invariant under multiplication by characters.

It was this corollary which led the author to hope that for $1<p<2$, $C v\left(L^{p}, L^{2}\right)$ would be the maximal subspace of $B_{2 \infty}^{n(1 / p-1)}$ invariant under translation.

The next result explains why it seems unlikely that there is equality in Theorem 3.2 for $q=2,1<p<2$. The result seems to say that the information in Theorem 3.2 follows from applying the definition of a multiplier to radial nonnegative functions. It also provides a kind of maximal estimate for multipliers involving "arbitrary" rectangles, as well as cubes.

Proposition. If $m \in \mathfrak{T}\left(L^{p}, L^{q}\right), q<2$, then for any rectangle $R$ in $R^{n}$,

$$
\left(\int_{R}|m(z)|^{q^{\prime}} d z\right)^{1 / q^{\prime}}<A\|m\||R|^{1 / p^{\prime}} .
$$


Proof. For $f \in \delta, F^{-1}(m \hat{f}) \in L^{q}$, and since $q<2$, it follows from the Hausdorff-Young inequality that

$$
\left(\int|m(z) \hat{f}(z)|^{q^{\prime}} d z\right)^{1 / q^{\prime}} \leqslant A\|m\|\|f\|_{p} .
$$

The inequality is also valid for any $f \in L^{p}$; in particular, for $\hat{f}=\chi_{R}$, the characteristic function of a rectangle, which gives the result.

Our next theorem is the affirmative part of the answer to an old question of Peetre [15]. Peetre asked whether a linear translation invariant operator which was such that for some $p, 1<p<\infty, T: \dot{B}_{p q}^{s} \rightarrow \dot{B}_{p q}^{s}, 0<s<\sigma, 1<q<\infty$, necessarily mapped $L^{p} \rightarrow L^{p}$. It was shown by Stein and Zygmund [19] and by Littman, McCarthy and Rivière [13] that the answer was negative. They did this by constructing (on the torus) an element of $B_{1 \infty}^{0}(T)$ which did not map $L^{p} \rightarrow L^{p}$. A similar example on $R^{n}$ was given by Rivière [18]. Our next result shows that a simple property of convoluteurs of $L^{p}$ not immediately shared by convoluteurs of $B_{p q}^{s}$ characterizes these operators.

THEOREM 3.3. Let $1<p<\infty$. A convolution operator $T: \dot{B}_{p q}^{s} \rightarrow \dot{B}_{p q}^{s}$, for some $s>0$ and $-s, 1 \leqslant q<\infty$, maps $L^{p} \rightarrow L^{p}$ iff $\forall h \in R^{n}, e^{i\langle x, h\rangle} T$ maps $\dot{B}_{p q}^{s} \rightarrow \dot{B}_{p q}^{s}$ and $\dot{B}_{p q}^{-s} \rightarrow \dot{B}_{p q}^{-s}$.

Proof. If $T: L^{p} \rightarrow L^{p}$, then by Theorem 1.13 of [7] $e^{i\langle x, h\rangle} T: L^{p} \rightarrow L^{p}$ for all $h$, hence, by Lemma 5 of [10], $e^{i x h} T: \dot{B}_{p q}^{s} \rightarrow \dot{B}_{p q}^{s}$, for all real $s$.

Conversely, if there is an $s$ such that $e^{i\langle x, h\rangle} T: \dot{B}_{p q}^{s} \rightarrow \dot{B}_{p q}^{s}, e^{i\langle x, h\rangle} T: \dot{B}_{p q}^{-s} \rightarrow$ $\dot{B}_{p q}^{-s}$ then, by interpolation, $e^{i\langle x, h\rangle} T: \dot{B}_{p q}^{0} \rightarrow \dot{B}_{p q}^{0}$, and since this holds for $q=\infty$, the result follows if we prove the next theorem.

THEOREM 3.4. $C v\left(L^{p}, L^{q}\right)=\left\{K \mid e^{i\langle x, h\rangle} K \in C v\left(\dot{B}_{p \infty}^{0}, \dot{B}_{q \infty}^{0}\right), \forall h \in R^{n}\right\}$, and $\|K\| \cong \sup _{h}\left\|e^{i\langle x, h\rangle} K\right\|$.

Proof. The inclusion $\subseteq$ follows by Lemma 5 of [10] and an application of Theorem 1.13 of Hormander [7].

The converse inclusion is proved in the following way. Let $e^{i\langle x, h\rangle} K \in$ $C v\left(\dot{B}_{p \infty}^{0}, \dot{B}_{q \infty}^{0}\right)$ for every $h \in R^{n}$ and let $M=\sup _{h}\left\|e^{i\langle x, h\rangle} K\right\|$. Take $f \in$ $L^{p}\left(R^{n}\right)$. Then $e^{i\langle x, h\rangle} f \in L^{p}\left(R^{n}\right) \subseteq \dot{B}_{p \infty}^{0}\left(R^{n}\right)$ for every $h$ and thus

$$
e^{i\langle x, h\rangle}(K * f)=e^{i<\cdot h\rangle} K * e^{i\langle\cdot, h\rangle} f \in \dot{B}_{q \infty}^{0}\left(R^{n}\right)
$$

and

$$
\dot{B}_{q \infty}^{0}\left(e^{i\langle x, h\rangle}(K * f)\right)<M \dot{B}_{p \infty}^{0}\left(e^{i\langle x, h\rangle} f\right)<M\|f\|_{p} .
$$

If $q>1$, we apply Theorem 1.2 and we see that

$$
\|K * f\|_{q}<A \sup _{h} \dot{B}_{q \infty}^{0}\left(e^{i\langle x, h\rangle}(K * f)\right)<M\|f\|_{p} \text {. }
$$

If $q=1, p=1$ and $C v\left(\dot{B}_{1 \infty}^{0}, \dot{B}_{1 \infty}^{0}\right)=\dot{B}_{1 \infty}^{0}$. Both sides are $\mathscr{T}\left(R^{n}\right)$ in this case. 
In the language of Peetre's book [17], while the imbedding $C_{p} \rightarrow \dot{B}^{0 \infty}\left(C_{p}\right)$ is not onto, we do have

$$
\pi\left(\dot{B}^{0 \infty}\left(C_{p}\right)\right)=C_{p} .
$$

The third application is to characterize the Fourier transform image of the Morrey spaces, in the only case for which you would expect a characterization.

THEOREM 3.5. If $1 \leqslant p \leqslant 2, f \in L^{p, \beta}, n>\beta>0$, then $e^{i\langle x, h\rangle} \hat{f} \in$ $\dot{B}_{p^{\prime} \infty}^{-\beta / p}\left(R^{n}\right)$ for every $h \in R^{n}$ with the norms bounded independent of $h$. For $p=2, \mathscr{F}: L^{2, \beta} \rightarrow \pi \dot{B}_{2 \infty}^{-\beta / 2}$ is an isomorphism.

Our fourth application is to obtain an improvement of a result proved by Herz [6] and Kellogg [11].

THEOREM 3.6. If $1<p<2, f \in L^{p}\left(R^{n}\right)$, then for any $\beta>0$ and for any $\lambda$,

$$
\lambda^{\beta}\left(\int_{\lambda}^{\infty}\left(t^{-p^{\prime} \beta} \int_{|z-y|<t}|\hat{f}(z)|^{p^{\prime}} d z\right)^{2 / p^{\prime}} t^{-1} d t\right]^{1 / 2}<A_{\beta}\|f\|_{p}
$$

Proof. For $f \in L^{p}\left(R^{n}\right), e^{i\langle x, h\rangle} f \in L^{p}\left(R^{n}\right)$ and thus $\tau_{h} \hat{f} \in K_{p^{\prime} 2}^{0}$ for each $h \in R^{n}$ and

$$
\sup _{h}\left\|\tau_{h} \hat{f}\right\| \leqslant \sup _{h}\left\|e^{i\langle x, h\rangle} f\right\|_{p}=\|f\|_{p}
$$

Finally we note that Theorem 1.4 provides a proof that there are no nonnegative elements in some of the Besov spaces.

THEOREM 3.7. If $1 \leqslant p \leqslant 2, \alpha \leqslant-n / p^{\prime}, 1 \leqslant q<\infty$ or $\alpha<-n / p^{\prime}$, $q=\infty$, and $\mu \geqslant 0, \mu \in \dot{B}_{p q}^{\alpha}\left(R^{n}\right)$, then $\mu=0$.

Proof. If $p>1, \alpha$ will be negative and we can use the fact that for $\alpha<0$,

$$
\left|W_{t} *\left(e^{i\langle x, h\rangle} \mu\right)\right| \leqslant W_{t} * \mu
$$

and, consequently,

$$
\dot{B}_{p q}^{\alpha}\left(e^{i\langle x, h\rangle} \mu\right) \leqslant \dot{B}_{p q}^{\alpha}(\mu) .
$$

It follows that for every $h, e^{i\langle x, h\rangle} \mu \in \dot{B}_{p q}^{\alpha}$, and by Theorem $1.4 \mu \equiv 0$.

If $p=1$, this argument will work for $q=\infty$, and for $1<q<\infty, \alpha<0$. It remains to consider $\alpha=0$.

For $q=1$, that $\mu=0$ follows since $\mu \in \dot{B}_{11}^{0}$ must be integrable with mean value 0 . If $1<q<\infty$, there is a $K$ such that $\mu * \mu * \cdots * \mu$ ( $K$ times) $\in$ $\dot{B}_{11}^{0}$, and thus $\mu * \cdots * \mu \equiv 0 \Rightarrow \mu=0$.

Our final application is to the study of multiplicative properties of homogeneous and inhomogeneous Besov spaces. Herz [0] has proved a number of such properties for homogeneous Besov spaces. It is not obvious that they 
carry over to inhomogeneous Besov spaces because of the added global constraint. Theorem 1.1 allows us to give an easy extension of these results to inhomogeneous spaces.

TheOREM 3.8. Suppose $0<\beta<\alpha \leqslant n(\beta \leqslant \alpha$ if $1 / q \geqslant 1 / p-\beta / n)$ and $p<n / \beta(p \leqslant n / \beta$ if $q=1), 1 \leqslant q<\infty, 1 \leqslant p<\infty$. Then $\dot{B}_{n / \alpha, 1}^{\alpha} B_{p q}^{\beta} \subseteq B_{p q}^{\beta}$ and $B_{n / \alpha, 1}^{\alpha} \dot{B}_{p q}^{\beta} \subset B_{p q}^{\beta}$.

Proof. Observe that $\pi X \cdot Y \subseteq \pi(X Y)$.

This result gives an improvement of some results of Peetre [17].

\section{REFERENCES}

1. A. Beurling, On two problems concerning linear transformations, Acta Math. 81 (1948), 239-255.

2. S. Campanato, Properties of a family of function spaces, Ann. Scuola Norm. Sup. Pisa 18 (1964), 137-160.

3. E. Fabes and U. Neri, Characterization of temperatures with initial data in BMO, Duke Math. J. 42 (1975), 725-734.

4. C. Fefferman and E. Stein, $H^{p}$ spaces of several variables, Acta Math. 129 (1972), 137-193.

5. T. Flett, Some elementary inequalities for integrals with application to Fourier transforms, Proc. London Math. Soc. (3) 29 (1974), 538-556.

6. C. Herz, Lipschitz spaces and Bernstein's theorem on absolutely convergent Fourier transforms, J. Math. Mech. 18 (1968), 283-324.

7. L. Hörmander, Estimates for translation invariant operators in $L^{p}$ spaces, Acta Math. 104 (1960), 93-140.

8. R. Johnson, Representation theorems and Fatou theorems for second-order linear parabolic partial differential equations, Proc. London Math. Soc. (3) 23 (1971), 325-347.

9. Lipschitz spaces, Littlewood-Paley spaces and convoluteurs, Proc. London Math. Soc. (3) 29 (1974), 127-141.

10.

11. C. N. Kellogg, An extension of the Hausdorff-Young theorem, Michigan Math. J. 18 (1971), 121-128.

12. P. Lax, Translation invariant spaces, Acta Math. 101 (1959), 163-178.

13. W. Littman, C. McCarthy and N. Rivière, $L^{p}$ multiplier theorems, Studia Math. 30 (1968), 193-217.

14. M. Murata, Asymptotic behavior at infinity of solutions of certain linear partial differential equations, J. Tokyo Univ. Fac. Sci. 23 (1976), 107-148.

15. J. Peetre, Espaces d'interpolation et théorème de Soboleff, Ann. Inst. Fourier (Grenoble) 16 (1966), 279-317.

16. On the theory of $\varrho^{p, \lambda}$ spaces, J. Functional Analysis 4 (1969), 71-87.

17. __ New thoughts on Besov spaces, Duke Univ. Math. Series, no. 1, Duke University, Durham, N. C., 1976.

18. N. Rivière, Classes of smoothness, the Fourier method (unpublished).

19. E. Stein and A. Zygmund, Boundedness of translation invariant operators on Hölder and $L^{p}$ spaces, Ann. of Math. (2) 85 (1967), 337-349.

Department of Mathematics, University of Maryland, College Park, Maryland 20742 